\title{
Domino shuffling on Novak half-hexagons and Aztec half-diamonds
}

\author{
Eric Nordenstam* \\ Fakultät für Mathematik, University of Vienna, Austria \\ eric.nordenstam@univie.ac.at \\ Benjamin Young ${ }^{\dagger}$ \\ Department of Mathematics, KTH, Stockholm, Sweden \\ benyoung@math.kth.se
}

Submitted: Apr 5, 2011; Accepted: Sep 1, 2011; Published: Sep 9, 2011

Mathematics Subject Classification: 82B20, 05A15

\begin{abstract}
We explore the connections between the well-studied Aztec Diamond graphs and a new family of graphs called the Half-Hexagons, discovered by Jonathan Novak. In particular, both families of graphs have very simple domino shuffling algorithms, which turn out to be intimately related. This connection allows us to prove an "arctic parabola" theorem for the Half-Hexagons as a corollary of the Arctic Circle theorem for the Aztec Diamond.
\end{abstract}

\section{Introduction}

In their groundbreaking paper [GV85] gave a method for counting families of non-intersecting lattice paths between two equinumerous sets of points. In their first example, the paths are between

$$
\left(0,-x_{i}\right) \text { and }(i,-i), 1 \leq i \leq n
$$

and are composed of unit-length steps up or to the right only. The number of such lattice paths is a Vandermonde determinant:

$$
\operatorname{det}\left[\left(\begin{array}{c}
x_{i} \\
j
\end{array}\right)\right]_{1 \leq i, j \leq n}=\prod_{1 \leq i<j \leq n} \frac{x_{j}-x_{i}}{j-i} .
$$

\footnotetext{
${ }^{*}$ Nordenstam was supported by the Austrian Science Foundation FWF, grant Z130-N13.

†Young was supported by grant KAW 2010.0063 from the Knut and Alice Wallenberg Foundation.
} 


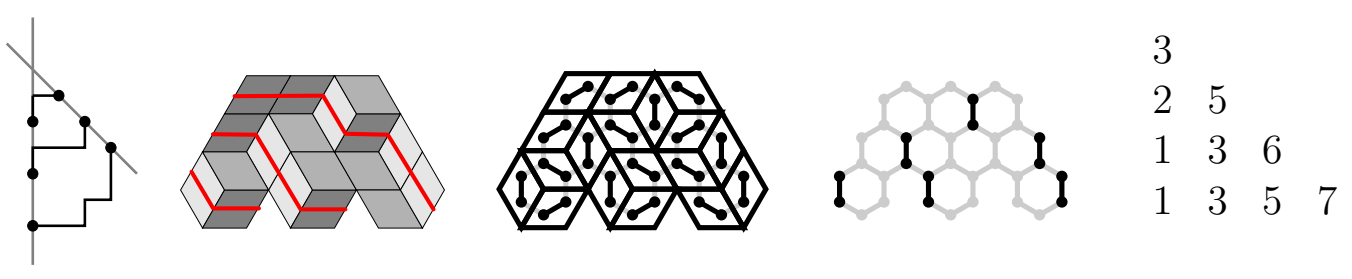

Figure 1: Five models which are in bijection: Non-intersecting lattice paths, pile of boxes, perfect matching and dual tiling, interlacing particle process, Staircase semistandard Young Tableau. All of these are order 3.

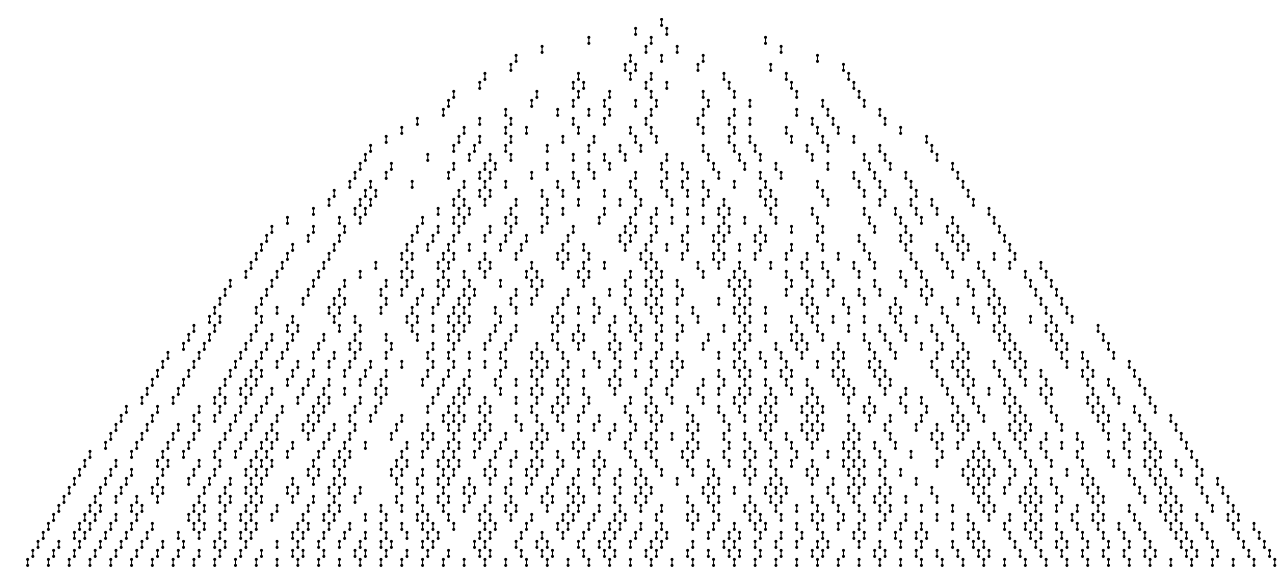

Figure 2: Order 100 half-hexagon, as an interlacing particle process

Indeed, these ideas were already present in the works of Lindström [Lin73] and KarlinMcGregor [KM59]; they are applicable far beyond the scope of these papers and form the enumerative-combinatorial cornerstone for many areas of modern mathematics.

We shall focus on a specific case of the above example of Gessel-Viennot. Jonathan Novak pointed out to us that when $x_{i}=2 i$, then the number of these paths is $2^{n(n+1) / 2}$, which is the same as the number of domino tilings of an Aztec diamond [EKLP92a]; he asked us for a bijection. We didn't find one, but we did find many amazing similarities between these two models. Namely, they have similar domino shuffles, and similar limit laws. A little further detective work turned up a family of subgraphs of the Aztec diamond, which we call Aztec half-diamonds, whose domino shuffling algorithm is identical, in a certain sense, to that on the half-hexagon. Our Aztec half-diamonds are similar but not identical to the Aztec half-diamond of [FF11].

We wish to thank Jonathan Novak for bringing this problem to our attention, as well as our colleagues Alexei Borodin, Dan Romik, for helpful conversations. This paper began at the 2010 program in Random Matrix Theory, Integrable Systems and Interacting Particle Processes at the Mathematical Sciences Research Institute in Berkeley, California; it was completed while B. Young was visiting Universitat Wien.

Several similar problems have been considered in the vast and ever-growing literature 
on the dimer problem, nonintersecting lattice paths, and the like. We mention some of them here.

- Okounkov and Kenyon [KO07] calculate limiting shapes for dimer models on portions of the hexagonal grid, under "polygonal" boundary conditions; they show that for a generic polygonal boundary with $3 d$ sides in which the edges appear in cyclic order, the limiting shape is an algebraic curve of degree $d$. They comment that some of these conditions can be relaxed; however it is not clear to us how to use these methods to handle the erratic bottom boundary of our half-hexagons.

- Di Francesco and Reshetikin [DFR09] study similar half-hexagonal shapes, but in which the long boundary is free; they obtain a variety of different limit shapes, none of which is the same as ours.

- Borodin and Ferrari [BF08] have a very general framework for studying dynamics on interlacing particle processes, including the Aztec diamond domino shuffle and many others. Though they do not handle this particular case, our model does fit into this framework; we shall hopefully carry out their analysis in a subsequent paper.

Very recently, Engstrom and Norén [EN11] have constructed a bijection such as the one we originally sought when we began this research. Their work largely uses ideas which were present in [EKLP92a], together with some tools from poset theory. We are now trying to develop a relationship between this bijection and our work.

\section{Bijective Combinatorics}

The Gessel-Viennot lattice path model is in bijection with a number of other combinatorial structures. Some of these bijections are "folklore" and all are well-known, but it is important to state briefly what they are, in order to establish terminology.

For the remainder of this section, fix the order $n \in \mathbb{Z}_{\geq 0}$.

\subsection{Non-intersecting lattice paths}

Let $\operatorname{NILP}(n)$ be the set of families of non-intersecting lattice paths which begin at the points $(0,-2 i)$ and end at the points $(i,-i)$, composed of steps of unit length in the directions of increasing $x$ and $y$. We get this from the example of [GV85] by taking $x_{i}=2 i$. The $n$th path from the top is of length $2 n$.

As mentioned above, it is easy to enumerate these families of paths using the method of Gessel-Viennot; we shall do this in Section 3.1.

\section{$2.2 \quad$ Lozenge tilings}

It is well known (see, for example, [GV89, Joh05b]), that any family of non-intersecting lattice paths on the square lattice, with fixed start and end points, is in bijection with 
lozenge tilings of a certain region of the triangular lattice. Here, a lozenge is a parallelogram composed of two adjacent equilateral triangles. The boundary of the region depends only upon the locations of the endpoints.

Definition 2.1 The regular triangular lattice $\mathcal{L}$ is the infinite planar graph whose vertices are the integer span of the vectors

$$
v=\left[\begin{array}{l}
1 \\
0
\end{array}\right] \quad w=\frac{1}{2}\left[\begin{array}{c}
1 \\
\sqrt{3}
\end{array}\right]
$$

and which has edges joining any two vertices which are unit distance apart. L subdivides $\mathbb{R}^{2}$ into unit equilateral triangles.

Let $R_{n} \subseteq \mathbb{R}^{2}$ be the union of the large trapezoid with corners

$$
\{n v, n w, n(w-v),-n v\}
$$

and the $n$ small trapezoids with corners

$$
\left\{\begin{array}{l}
(2 i-n+1) v+v \\
(2 i-n+1) v-v \\
(2 i-n+1) v-w \\
(2 i-n+1) v+v-w
\end{array} \mid i=0, \ldots, n-1\right\}
$$

See Figure 1, pictures 2 and 3, for a graphical representation of $R_{3}$. Let $\mathrm{LT}(n)$ be the set of lozenge tilings of $R_{n}$. To obtain an element of $\operatorname{LT}(n)$ given an element of $\operatorname{NILP}(n)$, first apply the affine transformation which takes the points

$$
\begin{aligned}
(0,-2 i) & \longmapsto(-n+2 i) v-\frac{1}{2} w, \\
(i,-i) & \longmapsto-n v+\left(i-\frac{1}{2}\right) w .
\end{aligned}
$$

Now the lattice steps are in directions $-v$ and $w-v$; each step begins and ends on the boundary of a triangle in $\mathcal{L}$ and traverses two of the triangles of $L$. Form a partial tiling by placing the corresponding lozenge over each step. The holes in this tiling can be covered uniquely by lozenges as well.

Observe that, in drawing this tiling, we have also drawn a pile of cubical boxes: each tile represents a visible face of a cube with faces parallel to the coordinate planes, viewed isometrically from the direction $(1,1,1)$.

\subsection{Perfect matchings on a half-hexagon}

Let $\mathcal{L}^{\vee}$ denote the planar dual of $\mathcal{L} . \mathcal{L}^{\vee}$ is the regular tiling of the plane with hexagons, sometimes called the honeycomb mesh, grid or lattice.

Definition 2.2 Let $R_{n}^{\vee}$ be the subgraph of $\mathcal{L}^{\vee}$ induced by those vertices which lie within $R$. We call $R_{n}^{\vee}$ the half-hexagon graph. Let $H H(n)$ denote the set of perfect matchings of $R_{n}^{\vee}$ 
There is a folklore bijection between $\mathrm{HH}(n)$ and $\mathrm{LT}(n)$. Suppose we are given an element $T$ of $\operatorname{LT}(n)$. Each lozenge in the tiling $T$ is composed of two triangles of $\mathcal{L}$, which

are dual to two vertices in $\mathcal{L}^{\vee}$. Join every such pair of vertices with an edge to obtain a matching in $\mathrm{HH}(n)$. The resulting matching is perfect (i.e. that every vertex is covered) by virtue of the fact that a tiling in $\mathrm{LT}(n)$ covers $R(n)$ completely.

\subsection{Interlacing particle process}

Observe that the vertical edges of $R_{n}^{\vee}$ are centered at the points

$$
\left\{\left(-n-\frac{1}{2}+j\right) v+(n-i) w \mid i=1, \ldots, n ; j=1, \ldots, i+n\right\} .
$$

In the above set, we call $i$ the row index and $j$ the position. Let $\pi \in \mathrm{HH}(n)$. Observe that the vertical edges in $\pi$ determine $\pi$ completely, subject to the following interlacing condition: if there are edges in positions $j$ and $j^{\prime}$ of row $i$, then there must be an edge in some position $j^{\prime \prime}$ of row $i-1$, with $j \leq j^{\prime \prime}<j$. Indeed, any collection of vertical edges which interlace in this manner determine a tiling in $\mathrm{HH}(n)$.

From $\pi$, then, we may construct an interlacing particle process

$$
\left\{(i, j) \mid\left(-n-\frac{1}{2}+j\right) v+(n-i) w \text { is the center of a vertical edge in } \pi\right\} \subset \mathbb{N}^{2} .
$$

Let $\operatorname{IPP}(n)$ denote the set of all such interlacing particle processes.

\subsection{Staircase tableaux}

Let $P$ be an element of $\operatorname{IPP}(n)$. Define the numbers $g_{i j}$ by

$$
P=\bigcup_{i=0}^{n-1}\left\{\left(i, g_{i j}\right) \mid 0 \leq j \leq i-1\right\}
$$

In other words, $g_{i j}$ is the position of the $j$ th vertical edge in row $i$ of the corresponding perfect matching in $H H(n)$. The interlacing conditions imply that

$$
g_{i j} \leq g_{i-1, j}<g_{i, j+1},
$$

in other words, that $\left(g_{i j}\right)$ is a Semistandard Young tableau of staircase shape [Sta99] with bottom row equal to $(1,3,5, \ldots, 2 n+1)$. We call these objects Staircase tableaux for short, and we write the set of all such as $\mathrm{ST}(n)$. Note also that the numbers

$$
h_{i j}=g_{i j}-j
$$

form a Gelfand-Tsetlin pattern (see $[$ Sta99, $(7.37)])$ with bottom row equal to $(0,1,2, \ldots, n)$. 


\section{Enumerative Combinatorics}

There are at least two easy ways to enumerate $\mathrm{HH}(n)$ by evaluating determinants. We shall introduce a third way as a consequence of the shuffling algorithm. Doubtless there are many others.

\subsection{Non-intersecting lattice path enumeration}

Gessel-Viennot [GV85] handle a slightly more general situation. Observe that there are $\left(\begin{array}{c}x_{i} \\ j\end{array}\right)$ up-right lattice paths from the point $\left(0,-x_{i}\right)$ to $(j,-j)$. Consequently, given $n$ integers $x_{1}<x_{2}<\cdots<x_{n}$, the number of families of nonintersecting lattice paths from $\left\{\left(0,-x_{i}\right\}\right.$ to $\{(j,-j)\}$ is given by (1). This determinant evaluation is given in [GV85]; it works because it is essentially a Vandermonde determinant. Putting $x_{i}=2 i$, we recover the endpoints of the paths for $\operatorname{NILP}(n)$, and we obtain

$$
|\operatorname{NILP}(n)|=2^{n(n+1) / 2} \text {. }
$$

\subsection{Staircase tableau enumeration}

An alternate easy enumeration, this time of ST $(n)$, goes through symmetric function theory. First we observe [Sta99, (7.37)] that because of the Gelfand-Tsetlin pattern interpretation, we have

$$
|\mathrm{ST}(n)|=s_{\lambda}(1, \ldots, 1)
$$

where $s_{\lambda}$ is a Schur function with $n+1$ arguments and $\lambda$ is the "staircase partition" $(1,2, \cdots, n+1)$. It is a consequence of the classical bialternant definition of the Schur function (see [Sta99, Chapter 7.15 and ex. 7.30] that

$$
s_{\lambda}\left(x_{1}, \ldots, x_{n+1}\right)=\prod_{1 \leq i<j \leq n}\left(x_{i}+x_{j}\right) .
$$

Putting all $x_{i}$ equal to 1 gives $|\mathrm{ST}(n)|=2^{n(n+1) / 2}$ as before. Indeed, performing the principal specialization $x_{i} \mapsto q^{i}$ gives a $q$-enumeration of $\mathrm{HH}(n)$, in which each element $\pi$ of $\mathrm{HH}(n)$ is assigned weight proportional to $q^{\operatorname{vol}(\pi)}$. Here, $\operatorname{vol}(\pi)$ denotes the integral of the height function of $\pi$. See [Ken04] for an introduction to the height functions of dimer models.

\section{Dynamics}

\subsection{The half-hexagon shuffle}

In this section, we will define dynamics on interlacing particles, called the half-hexagon shuffle, which takes the form of a random map from $\mathrm{HH}(n)$ to $\mathrm{HH}(n+1)$. The procedure is easiest to describe and implement on the staircase tableaux $\mathrm{ST}(n)$, and it is easiest to illustrate on the half-hexagon perfect matchings $\mathrm{HH}(n)$ (see Figure 3). 


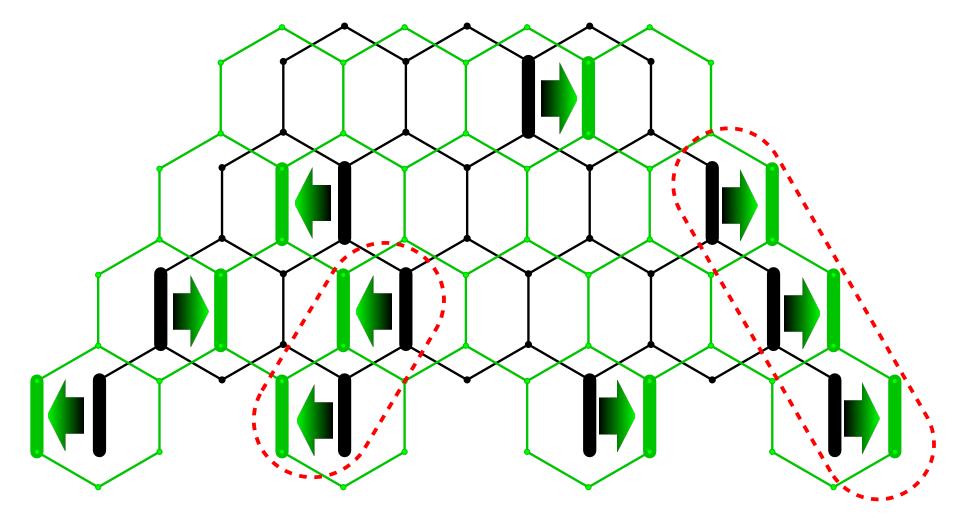

Figure 3: Domino shuffling on the half-hexagon. Groups of edges which are forced to move together, in order to maintain the interlacing conditions, are circled.

The half-hexagon shuffle constructs a perfect matching $\pi^{\prime}$ on $R^{\vee}(n+1)$ from $\pi$. It is not a deterministic process: one has to make a series of fair coin tosses to determine $\pi^{\prime}$. As it turns out, these coin tosses provide a direct explanation of the fact that the cardinality of $H H(n)$ is a power of two.

Algorithm 4.1 (Domino shuffling for the half-hexagon)

Input:

$\left(g_{i j}\right)$, a staircase tableau in $S T(n)$,

$\left(\xi_{i j}\right)$, independent Bernoulli 0-1 variables.

Output:

$\left(h_{i j}\right)$, a staircase tableau in $S T(n+1)$.

for $i$ from 0 to $n$ :

for $j$ from 0 to $i$ :

if $j<i$ and $g_{i, j}=h_{i-1, j}$ then:

$h_{i, j} \leftarrow g_{i, j}$

else if $j>0$ and $g_{i, j}=h_{i-1, j-1}$ then:

$$
h_{i, j} \leftarrow g_{i, j}+1
$$

else

$$
h_{i, j} \leftarrow g_{i, j}+\xi_{i, j}
$$

for $j$ from 0 to $n$ :

$$
h_{n+1, j}=2 j+1
$$

It is easier to illustrate this algorithm acting on the half-hexagon (see Figure 3 ) though slightly harder to describe. Begin with a perfect matching $\pi$ on $H H(n)$ (specified by its vertical edges). Observe that it is possible to add, deterministically, a row of $n+1$ vertical edges, appearing every second edge, to the bottom of $\mathcal{L}^{\vee}$, in such a way as to maintain the 
interlacing condition. Further, it is possible to superimpose the next-larger half-hexagon $R^{\vee}(n+1)$ on this graph, in such a way that each vertical edge is in the center of a hexagon of $R^{\vee}(n+1)$.

Working from the top of the graph to the bottom, each vertical edge in $\pi$ jumps either left or right onto the nearest vertical edge in the same row of $R^{\vee}(n+1)$. These jumps happen independently at random, according to the result of a fair coin toss, with the following exceptions:

- if moving an edge left would violate the interlacing condition with the new row above, then the edge moves right with probability 1.

- if moving an edge right would violate the interlacing condition with the new row above, then the edge moves left with probability 1.

We invite the reader to check the preceding procedure is the same as Algorithm 4.1.

One does need to check that the output of Algorithm 4.1 is always a staircase tableau, i.e. to verify that the interlacing conditions hold. This is done inductively on the row $i$, together with a checking that the deterministic row $n+1$ interlaces with row $n$.

\subsection{Preserving the uniform distribution}

In this section, we argue that the domino shuffle described above preserves the uniform distribution. We first introduce the time reversal of Algorithm 4.1.

Algorithm 4.2 (Time-reversed domino shuffle) (Time-reversed domino shuffling for the half-hexagon)

Input:

$\left(g_{i j}\right)$, a staircase tableau in $S T(n+1)$,

$\left(\xi_{i j}\right)$, independent Bernoulli 0-1 variables.

Output:

$\left(h_{i j}\right)$, a staircase tableau in $S T(n)$.

for $j$ from 0 to $n$ :

$h_{n, j}=2 j+1$

for $i$ from $n-1$ down to 0 :

for $j$ from 0 to $i$ :

if $g_{i, j}=h_{i+1, j}$ then:

$h_{i, j} \leftarrow g_{i, j}$

else if $g_{i, j}=h_{i+1, j+1}$ then:

$h_{i, j} \leftarrow g_{i, j}-1$

else

$$
h_{i, j} \leftarrow g_{i, j}-\xi_{i, j}
$$


On the half hexagon, this algorithm does the following: the $(n+1)$ st row of vertical edges is dropped altogether; the $n$th row jumps deterministically to positions $0,2, \ldots$, $n$. Then, working from the bottom to the top, edges jump left or right with probability $\frac{1}{2}$, except that edges are sometimes forced to move in order to interlace with edges in the row below.

Definition 4.3 Let $\mathbb{C H H}(n)$ denote the vector space whose orthonormal basis is indexed by the elements of $H H(n)$. Let $\langle\cdot, \cdot\rangle_{n}$ denote the inner product which makes this an orthonormal basis.

Let $P\left(\pi \rightarrow \pi^{\prime}\right)$ be the probability that Algorithm 4.1 produces output $\pi^{\prime}$ when given input $\pi$.

Let $\psi: \mathbb{C H H}(n) \rightarrow C H H(n+1)$ be the linear map for which

$$
\left\langle\psi(\pi), \pi^{\prime}\right\rangle=P\left(\pi \rightarrow \pi^{\prime}\right) .
$$

Lemma 4.4 The maps $\psi$ and $\psi^{\prime}$ are adjoint to each other. That is, if $\pi \in H H(n-1)$ and $\psi \in H H(n)$, then

$$
\left\langle\psi \pi, \pi^{\prime}\right\rangle=\frac{1}{2^{n}}\left\langle\pi, \psi^{\prime} \pi^{\prime}\right\rangle .
$$

Proof. Algorithms 4.1 and 4.2 move the edges either randomly (steps 4.1 and 4.2) or deterministically (steps 4.1, 4.1, 4.2 and 4.2). We call the random moves free and the deterministic moves forced, because all of the deterministic moves occur in blocks, precipitated by a preceding free move. Figure 3 shows an instance of the shuffling algorithm, with blocks of forced moves circled. In this terminology, we have

$$
\begin{aligned}
& \left\langle\psi \pi, \pi^{\prime}\right\rangle=2^{-\#\left\{\text { free choices in } \pi \stackrel{\psi}{\rightarrow} \pi^{\prime}\right\}}
\end{aligned}
$$

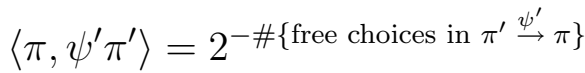

Let $S$ be all of the free choices in $\pi \stackrel{\psi}{\rightarrow} \pi^{\prime}$; and let $S^{\prime}$ be the set of all free choices which do not force any edges in row $n$ of $\pi$ to move. Since there are $n$ edges in the bottom row, $|S|=\left|S^{\prime}\right|+n$.

Let $e_{i}$ be one of the edges in $E$ in row $i$, and suppose that in passing from $\pi$ to $\pi^{\prime}$. Say that $e_{i}$ forces the movement of edges $e_{i+1}, \ldots, e_{i^{\prime}}$, in successive rows. Observe, then, that the time-reversed algorithm $\psi^{\prime}$, in passing from $\pi^{\prime}$ to $\pi$, sees a free choice at edge $e_{i^{\prime}}$ which forces edges $e_{i}, \ldots, e_{i+1}$ to move. Moreover, $\pi^{\prime}$ moves row $n$ deterministically (and then deletes it) so these are never free choices. As such, the free choices in $\pi^{\prime} \stackrel{\psi^{\prime}}{\rightarrow} \pi$ are in bijection with $S^{\prime}$.

$$
\left\langle\psi \pi, \pi^{\prime}\right\rangle=2^{-|S|}=2^{-\left|S^{\prime}\right|-n}=2^{-n}\left\langle\pi, \psi^{\prime} \pi^{\prime}\right\rangle
$$

Definition 4.5 Let $\mu_{n} \in \mathbb{C} H H(n)$ denote the vector corresponding to the uniform probability distribution on $\mathrm{HH}(n)$ :

$$
\mu_{n}=\frac{1}{|H H(n)|} \sum_{\pi \in H H(n)} \pi .
$$


Proposition 4.6 Domino shuffling preserves the uniform distribution:

$$
\psi \mu_{n-1}=\mu_{n}
$$

Proof. This is a straightforward computation:

$$
\begin{aligned}
\psi \mu_{n-1} & =\sum_{\pi \in \mathrm{HH}(n-1)} \sum_{\pi^{\prime} \in \mathrm{HH}(n)}\left\langle\psi \pi, \pi^{\prime}\right\rangle \pi^{\prime} \\
& =\frac{1}{2^{n}} \sum_{\pi^{\prime} \in \mathrm{HH}(n)} \pi^{\prime}\left(\sum_{\pi \in \mathrm{HH}(n-1)}\left\langle\pi, \psi^{\prime} \pi^{\prime}\right\rangle\right) \\
& =\frac{1}{2^{n}} \sum_{\pi^{\prime} \in H H(n)} \pi^{\prime} .
\end{aligned}
$$

In the second line, the bracketed sum is equal to one for any $\pi^{\prime}$ because $\psi^{\prime}$ is stochastic. The latter vector is proportional to $\mu_{n}$, and is thus equal to $\mu_{n}$ because the coordinates of both vectors sum to one.

Note that it follows from this proof that $|H H(n)|=2^{n}|H H(n-1)|$, which provides a new derivation of the fact that $|H H(n)|=2^{n(n+1) / 2}$.

\section{Review: the Aztec diamond}

The Aztec Diamond of order $n$ (which shall be denoted $A_{n}$ ) is a certain shape in the plane that can be covered with $2 \times 1$ rectangles, so called dominoes, in $2^{n(n+1) / 2}$ ways. More precisely, $A_{n}$ consists of the union of all squares whose corners have integer coordinates, whose sides are parallel to the coordinate axes and whose interiors are contained in $\left\{(x, y) \in \mathbb{R}^{2}:|x|+|y|<n+1\right\}$. The model was introduced in [EKLP92a, EKLP92b] in the study of Alternating sign matrices and a good survey is [Joh05a]. In this section we give a short review of the results we need about this model with citations.

One way to study the random tilings in this model is to introduce a certain particle process, see [Joh05a, Nor10] and also Figure 4 for details. In short, the lattice on which the dominos are placed is colored like a chessboard, and all dominoes whose bottommost or rightmost square is dark is represented by a particle. The tiling does uniquely determine the positions of the particles. The converse is only true modulo the fact that there will be $2 \times 2$-rectangles where two horizontal dominoes can be switched for two vertical ones or the other way around. Particle configurations coming from a tiling of $A_{n}$ are in bijection with Alternating sign matrices and this is the original motivation for studying this tiling model. However, the measure on particle configurations that is induced by uniform measure on all possible tilings is not the same as uniform measure on all Alternating sign matrices (it is, rather, connected to the 2-enumeration of alternating sign matrices, see [EKLP92a]).

In a tiling of $A_{n}$ there are $\left(\begin{array}{c}n+1 \\ 2\end{array}\right)$ particles on $n$ rows, see Figure 4 . Along the line $y=1$ there is a single particle, along line 2 there are two, etc. Let $x_{j}^{i}$ be the position of the $j$ th 


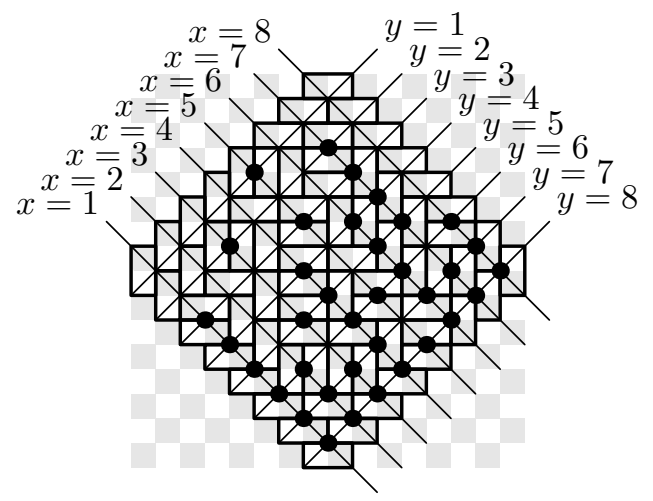

Figure 4: Particle processes associated with domino tilings of an Aztec diamond. The underlying lattice is colored like a chessboard and all dominoes whose bottommost or rightmost square is dark is represented by a particle.

particle on line $y=i$. Observe that the particles interlace in the sense that

$$
x_{j}^{i+1} \leq x_{j}^{i} \leq x_{j+1}^{i+1} \quad \text { for all } 1 \leq j \leq i
$$

this is similar, but not the same, as the interlacing conditions for the half-hexagon particle process in Section 2.4.

There is an algorithm, called the shuffing algorithm, that can be used to construct a random tiling and is described in great depth in each of [EKLP92b, JPS98, Pro03]. The procedure starts with a tiling of $A_{n}$; one moves the dominos of the tiling about in a certain way, decided by a certain number of coin flips, producing a tiling of $A_{n+1}$. If one starts with the empty tiling of $A_{0}$, performs this algorithm $n$ times, tossing fair coins all the way, then one ends up with a sample from the uniform distribution of all possible tilings.

The idea of [Nor10] is to look at the positions of the aforementioned particles under the evolution of this algorithm. It turns out that the particles are not glued to the tiles. The dynamics of these particles are as follows. In the following all $\gamma_{j}^{i}(t)$ for $i, j, t=1,2$, $\ldots$, are independent Bernoulli random variables, that is one with probability $\frac{1}{2}$ and zero otherwise.

It turns out that the first particle performs the simple random walk

$$
x_{1}^{1}(t)=x_{1}^{1}(t-1)+\gamma_{1}^{1}(t) .
$$

The particle $x_{1}^{2}$ performs a simple random walk with a reflecting boundary. More precisely, while $x_{1}^{2}(t)<x_{1}^{1}(t)$ it performs a random walk independently of $x_{1}^{1}$, at each time either staying or adding one with equal probability. However, when there is equality, $x_{1}^{2}(t)=$ $x_{1}^{1}(t)$, it is pushed forward by that particle. In order to represent this as a formula, we subtract one if the particle attempts to jump past $x_{1}^{1}$.

$$
x_{1}^{2}(t)=x_{1}^{2}(t-1)+\gamma_{1}^{2}(t)-\mathbf{1}\left\{x_{1}^{2}(t-1)+\gamma_{1}^{2}(t)=x_{1}^{1}(t-1)+1\right\}
$$


Since the diamond is symmetric under the flip of $x$ to $n+1-x$ we can get the recursion for $x_{2}^{2}$ by substituting

$$
\begin{array}{rlrl}
x_{1}^{2}(t) & \mapsto t+1-x_{2}^{2}(t), & x_{1}^{2}(t-1) \mapsto t-x_{2}^{2}(t-1), \\
\gamma_{1}^{2}(t) \mapsto 1-\gamma_{2}^{2}(t), & x_{1}^{1}(t-1) \mapsto t-x_{1}^{1}(t-1) .
\end{array}
$$

Simplifying gives

$$
x_{2}^{2}(t)=x_{2}^{2}(t-1)+\gamma_{2}^{2}(t)+\mathbf{1}\left\{x_{2}^{2}(t-1)+\gamma_{2}^{2}(t)=x_{1}^{1}(t-1)\right\} .
$$

The same pattern repeats itself evermore.

$$
\begin{aligned}
x_{1}^{j}(t)=x_{1}^{j}(t-1)+\gamma_{1}^{j}(t)-1\left\{x_{1}^{j}(t-1)+\gamma_{1}^{j}(t)\right. & \left.=x_{1}^{j-1}(t-1)+1\right\} \\
x_{j}^{j}(t)=x_{j}^{j}(t-1)+\gamma_{j}^{j}(t)+1\left\{x_{j}^{j}(t-1)+\gamma_{j}^{j}(t)\right. & \left.=x_{j-1}^{j-1}(t-1)\right\} \\
x_{i}^{j}(t)=x_{i}^{j}(t-1)+\gamma_{i}^{j}(t)-\mathbf{1}\left\{x_{i}^{j}(t-1)+\gamma_{i}^{j}(t)\right. & \left.=x_{j}^{j-1}(t-1)+1\right\} \\
+\mathbf{1}\left\{x_{i}^{j}(t-1)+\gamma_{i}^{j}(t)\right. & \left.=x_{j-1}^{j-1}(t-1)\right\} .
\end{aligned}
$$

with initial conditions $x_{i}^{j}(j)=i$ for $1 \leq i \leq j$.

In the analysis [Nor10] of the asymptotics of the domino shuffling algorithm, it turns out to be quite inconvenient that the $n$ particles on level $n$ are not created until time $n$. A simple change of variables will fix this. Let

$$
X_{i}^{j}(t)=x_{i}^{j}(t+i)
$$

for $1 \leq i \leq j$ and $t=1,2, \ldots$. We mention this here for a completely different reason: Rewriting the above recursion formulas in terms of the variables $\left(X_{i}^{j}\right)_{1 \leq i \leq j}$ gives the same recursion as is implemented by Algorithm 4.1 above (though with a different initial condition, see Section 6.1).

\section{The Aztec Half-Diamond}

Recall the definition of the Aztec diamond $A_{n}$ in Section 5. The Aztec half-diamond $H_{n}$ of order $n$ is a certain subregion of $A_{n}$. More precisely, for $n$ even,

$$
H_{n}=\left\{(x, y) \in A_{n}: y \geq 2\lfloor x / 2\rfloor\right\}
$$

and, for $n$ odd,

$$
H_{n}=\left\{(x, y) \in A_{n}: y+1 \geq 2\lfloor(x+1) / 2\rfloor\right\} .
$$

Though the definition as stated is a bit cryptic, the Figure 6 should make this quite clear. 


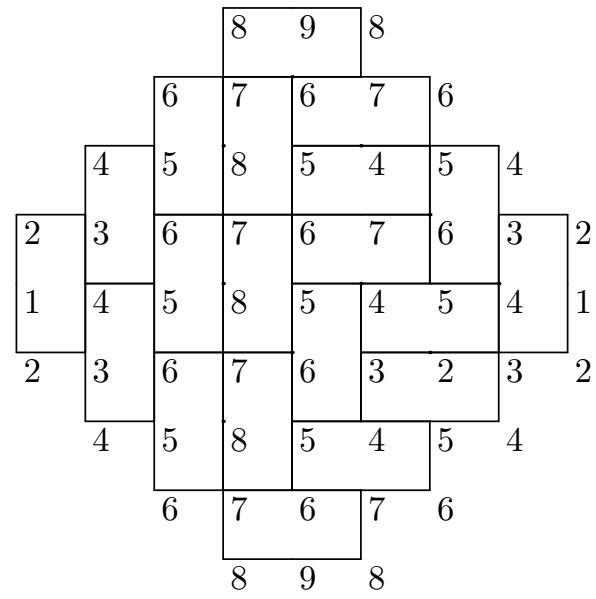

Figure 5: The height function is defined on the lattice on which the corners of the dominoes line up.
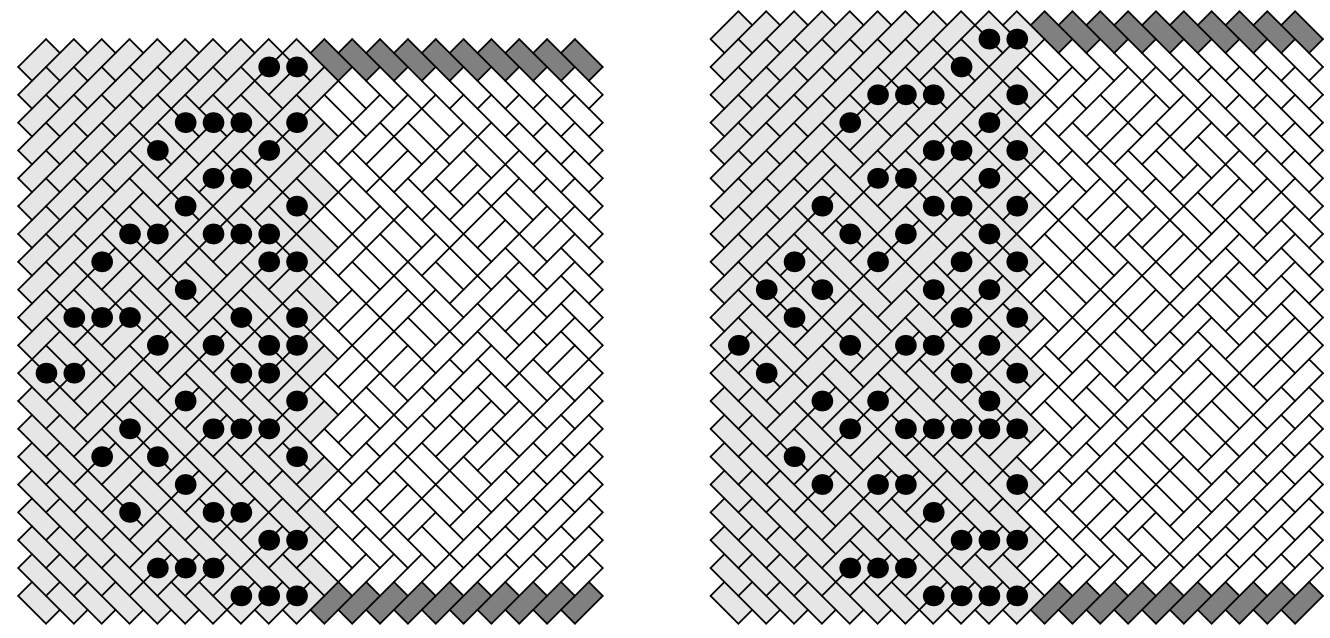

Figure 6: Two Aztec half-diamonds make an Aztec diamond. The left picture shows half-diamonds of orders 18 and 20; the right picture orders 19 and 21. The interlacing particle process is also shown. The right picture is the domino shuffle of the left. 

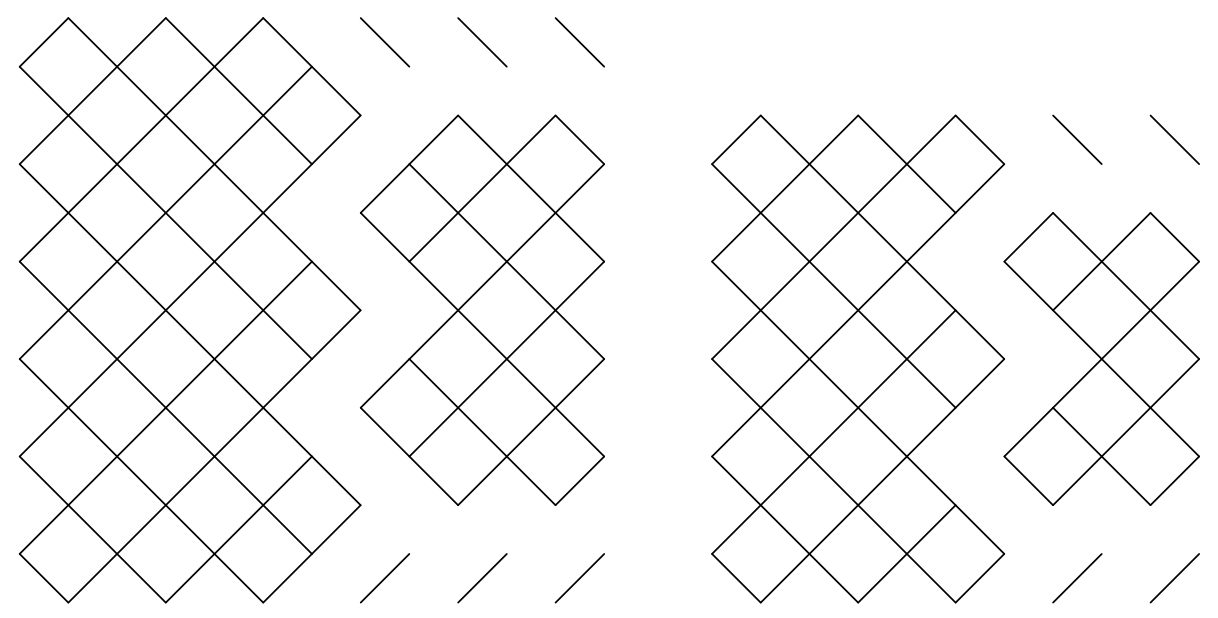

Figure 7: Edge weights for embedding two Aztec-half-diamonds in an Aztec diamond. Left: even-order case; Right: odd-order case. Unmarked edges get weight $\varepsilon \rightarrow 0$, while marked edges get weight 1 .

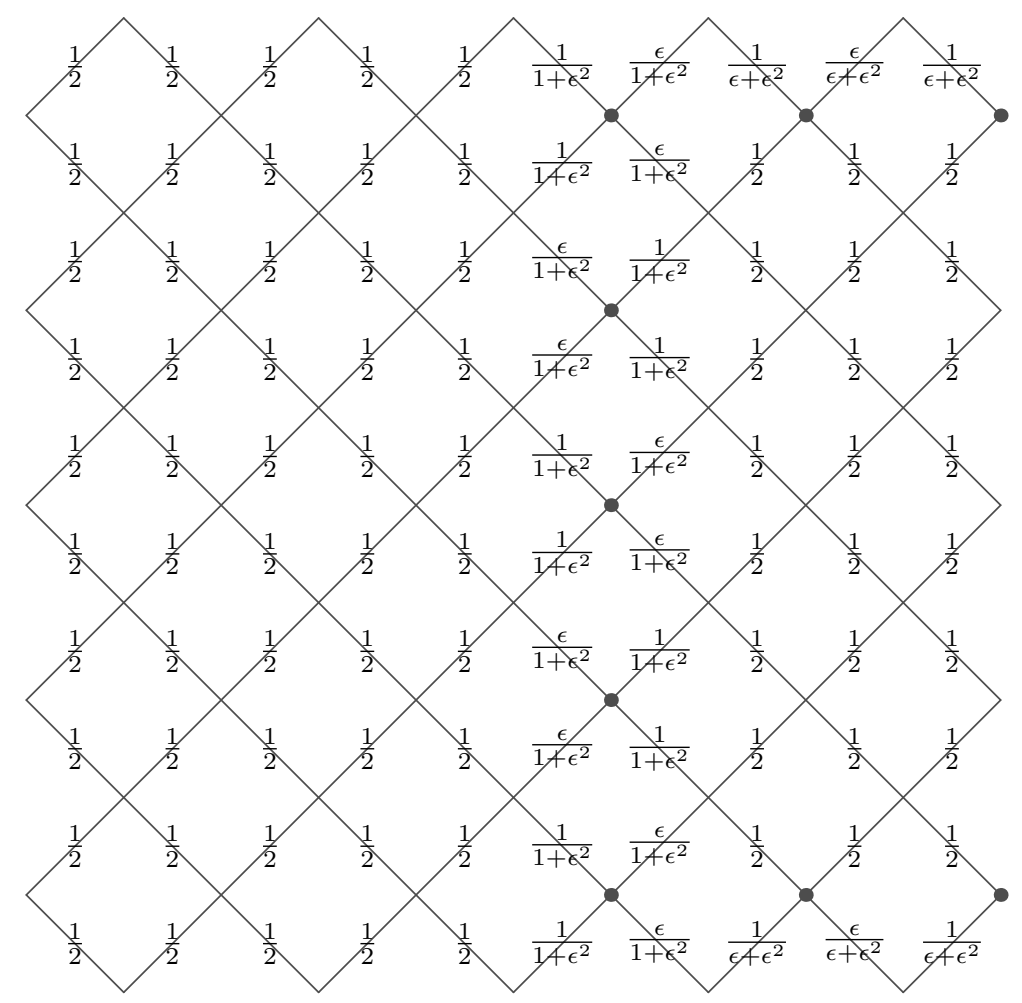

Figure 8: Weights computed by applying generalized domino shuffling to the even-order weights in Figure 7 . They give the same probability measure as the odd-order weights in Figure 7 upon taking $\varepsilon \rightarrow 0$ : perform gauge transformations at the marked points. 


\subsection{Dynamics: Domino shuffling}

The Aztec Half-Diamonds also have a domino shuffling algorithm: it arises in a somewhat surprising manner from an algorithm of Propp's [Pro03], "generalized domino shuffling", for generating random perfect matchings on certain planar bipartite graphs $G$. The strategy is to embed the graph $G$ into an Aztec diamond of sufficiently large order $n$, and then compute a certain series of probabilities $P_{j}^{i}(m)$ where $1 \leq m \leq n$ and $1 \leq i, j \leq$ $m$. The probabilities, in turn are given in terms of edge weights $w_{m}(e)$ on the order- $m$ Aztec diamonds for $1 \leq m \leq n$; these probabilities are precomputed by the algorithm. (this is perhaps an overly concise summary, but both the manner of embedding and the means of computation are described explicitly enough in [Pro03] to allow computer implementation). To generate the perfect matching, one then simply runs the domino shuffling algorithm, with the following modification: make $\xi_{j}^{i}(m)$ a Bernoulli random variable which takes the value 1 with probability $P_{j}^{i}(m)$. The final tiling of the Aztec Diamond of order $n$, restricted to the embedded copy of $G$, turns out to be uniformly random; the intermediary tilings of the smaller Aztec diamonds may be discarded.

The edge weights for producing uniform random tilings of the Aztec Half-diamonds are shown in Figure 7. The marked edges get weight 1, whereas the unmarked edges get a small positive weight $\varepsilon$ tending to zero (it is possible, but more awkward, to allow for weight-zero edges in the algorithm; see [Ciu98, JDLRV06]).

Definition 6.1 Let $z_{i}^{j}(t)$ denote the particle process given by generalized domino shuffling with weights $w_{t}(e)$ (defined analagously to the process $x_{i}^{j}$ of the Aztec diamond).

Proposition 6.2 The constant-time sections $t=t_{0}$ of $z_{i}^{j}(t)$, restrict to the uniform distribution on domino tilings of the order- $t_{0}$ Aztec half-diamond.

Proof. Suppose inductively that $w_{t}(e)$ are as shown in figure 7 , so as to produce the uniform distribution on Aztec half-diamonds. Apply Propp's rules to compute a new weight function $w^{\prime}(e)$. We need to check that $w^{\prime}(e)$ gives the same probability measure on dimer configurations as $w_{t-1}(e)$ in the limit $\varepsilon \rightarrow 0$.

We next apply a standard trick in the theory of the dimer model [KO07].

Definition 6.3 A gauge transformation of the edge weights $W(e)$ is a new set of edge weights, obtained by performing the following operation iteratively, one or more times: Choose a vertex $v$ of the graph and a constant $c$, and replace $W(e)$ with $c W(e)$ for every edge e incident to $v$.

A gauge transformation has no effect on the corresponding Boltzmann distribution on perfect matchings, since precisely one such edge $e$ is present in every perfect matching. Observe that by performing gauge transformations at the black dots in Figure 8, we can transform the weighting $w_{t-1}(e)$ into that of $w_{t-1}(e)$, up to a scalar factor of $1 / 2$, in the limit $\epsilon \rightarrow 0$. We conclude that the constant-time slice $t=t_{0}$ of the particle process $z_{i}^{j}(t)$ gives a uniform tiling of the Aztec half-diamond of order $t_{0}$. 
As such, if we remember the intermediary perfect matchings instead of discarding them, Propp's algorithm gives rise to a domino shuffle for the Aztec half-diamond in our sense: a random, locally defined map which increases the size of the half-diamond but preserves the uniform distribution. Indeed, this procedure is exactly the same as ordinary domino shuffling everywhere except the center line when $n$ is even; at these times, the particles in the center are forced to jump to equally spaced positions. This is equivalent to imposing the condition

$$
x_{i}^{m+1}(2 m+1)=2 i
$$

for $1 \leq i \leq m$.

\subsection{Height functions and limit shape}

It is possible [Thu90] to associate a discrete surface in $\mathbb{R}^{3}$, called a height function, to any domino tiling of the plane. More precisely, the height function $h: \mathbb{Z}^{2} \rightarrow \mathbb{Z}$, where the domain is the square grid whose vertices coincide with the corners of the dominos and the centers of their edges.

Definition 6.4 Let $T$ be a domino tiling of the region $R$. Then $h: \mathbb{Z}^{2} \rightarrow \mathbb{Z}$ is a height function for $T$ if, whenever $x+y \equiv 0(\bmod 2)$ and $(x, y)$ is in $R$, then

- $h(x, y)=h(x, y+1)+1$ if the edge from $(x, y)$ to $(x, y+1)$ does not cross a domino in $T$;

- $h(x, y)=h(x+1, y)-1$ if the edge from $(x, y)$ to $(x+1, y)$ does not cross a domino in $T$.

Note that $h$ determines $T$ uniquely, and that two height functions for $T$ differ only by a constant.

This definition coincides with those in [Thu90, CKP01] and, In the case where the region $R$ is an Aztec Diamond, this definition appears in [EKLP92a], where it is closely related to the height function for an alternating sign matrix.

The reader should be advised that there are closely related concepts in the literature called relative height functions [KO07, KOS06], edge-placement probabilities and onepoint functions for the particle process [Ken97, Pro03].

Fix a region $R$, a tiling $T$ of $R$, and a height function $h$ for $T$. Since no tiles ever cross the boundary of $R$, the restriction of $h$ to $\partial R$ is independent of $T$. Indeed, $\left.h\right|_{\partial R}$ can even be computed without specifying $T$ at all.

Definition 6.5 The function $\left.h\right|_{\partial R}$ is called a boundary height function.

Proposition 6.6 (See [Thu90, Section 4]) $R$ possesses a domino tiling if and only if $R$ has a well-defined boundary height function. 
Once it became possible to generate uniformly random tilings of large Aztec diamonds, it became immediately obvious that all such tilings have the same "shape". It was first shown in [JPS98] that a typical tiling of a large Aztec diamond has all of its disorder concentrated in a circular region, where the circle is tangent to all four sides of the diamond; the tiling is frozen close to the four corners.

In fact, more is true: the height function of a uniformly random tiling of an Aztec diamond tends to the following explicit limit. This limit seems possible to do using the correlation kernel for the Aztec Diamond, as defined in[Joh05a]; however, the first explicit derivation seems to be in [CEP96]. We use a later description of the asymptotic height function, given in [Rom09]. The coordinates are slightly different: in the following theorem, $h_{i, j}^{*}$ represents a rescaled height function of an order $n$ Aztec diamond, where the domain is to $[0,1] \times[0,1]$ and the range is rescaled to $[0,1]$. Indeed, for the remainder of this section we will work in these coordinates.

Theorem 6.7 (Theorem 11' in [Rom09]) Define

$$
\begin{aligned}
Z(x, y)= & \frac{2}{\pi}\left[(x-1 / 2) \arctan \left(\frac{\sqrt{\frac{1}{4}-(x-1 / 2)^{2}-(y-1 / 2)^{2}}}{1 / 2-y}\right)\right. \\
& +\frac{1}{2} \arctan \left(\frac{2(x-1 / 2)(1 / 2-y)}{\sqrt{\frac{1}{4}-(x-1 / 2)^{2}-(y-1 / 2)^{2}}}\right) \\
& \left.-(1 / 2-y) \arctan \left(\frac{x-1 / 2}{\sqrt{\frac{1}{4}-(x-1 / 2)^{2}-(y-1 / 2)^{2}}}\right)\right] .
\end{aligned}
$$

and define

$$
G[x, y]= \begin{cases}x+y & 0 \leq x \leq \frac{1-2 \sqrt{y(1-y)}}{2} \\ x+Z(x, y) & \frac{1-2 \sqrt{y(1-y)}}{2}<x<\frac{1+2 \sqrt{y(1-y)}}{2} \\ x-y & \frac{1+2 \sqrt{y(1-y)}}{2} \leq x \leq 1\end{cases}
$$

Then as $n \rightarrow \infty$ we have the convergence in probability

$$
\max _{0 \leq i, j \leq n}\left|\frac{h_{i, j}^{*}{ }^{n}}{n}-G(i / n, j / n)\right| \underset{n \rightarrow \infty}{\stackrel{\mathbb{P}}{\longrightarrow}} 0 .
$$

In comments after Equation (15), [Rom09] observes that

$$
G[x, 1 / 2]=\frac{1}{2}
$$

that is to say, that the limiting height function is constant across the center of the Aztec Diamond. (It is also possible to observe this directly: the Aztec diamond has a reflection symmetry in the line $y=1 / 2$ which leaves the uniform measure invariant, but which 
negates height functions up to a constant). This observation is quite important for our purposes: it means that the limit shape for Aztec Diamonds coincides with the boundary height function for Aztec Half-Diamonds.

If we replace the Aztec diamonds with a sequence of regions $R_{n}$ approximating a given region $R$, in such a way that the rescaled boundary height functions also tend to a limiting boundary height function on $R$, then there is still a unique limiting shape and a variational principle (though in general there is no reason to expect this limiting shape to be as nice as a circle!)

Theorem 6.8 ([CKP01, Theorem 1.1] Let $R^{*}$ be a region in $\mathbb{R}^{2}$ bounded by a piecewise smooth, simple closed curve $\partial R^{*}$. Let $h_{b}: \partial R^{*} \rightarrow \mathbb{R}$ be a function which can be extended to a function on $R^{*}$ with Lipschitz constant at most 2 in the sup norm. Let $f: R^{*} \rightarrow \mathbb{R}$ be the unique such Lipschitz function maximizing the entropy functional Ent $(f)$, subject to $\left.f\right|_{\partial R^{*}}=h_{b}$.

Let $R$ be a lattice region that approximates $R^{*}$ when rescaled by a factor of $1 / n$, and whose normalized boundary height function approximates $h_{b}$. Then the normalized height function of a uniformly random tiling of $R$ approximates $f$, with probability tending to 1 as $n \rightarrow \infty$.

In the above theorem, $\operatorname{Ent}(f)$ is given by

$$
\operatorname{Ent}(h)=\frac{1}{\operatorname{area}\left(R^{*}\right)} \iint_{R^{*}} \operatorname{ent}\left(\frac{\partial h}{\partial x}, \frac{\partial h}{\partial y}\right) d x d y,
$$

and ent $\left(\frac{\partial h}{\partial x}, \frac{\partial h}{\partial y}\right)$ is a certain explicit function of the gradient of the surface $h$; see [CKP01] for further details. For us, the most important point is that the asymptotic number of tilings depends only upon the asymptotic height function $g$, not upon the precise nature of the boundary of $R$; moreover this dependence is local. As such, an immediate consequence of Theorem 6.8 is the following:

Lemma 6.9 Let $S \subseteq R$ be regions. Let $h_{\partial R}$ be a boundary height function on $\partial R$, and let $f: R \rightarrow \mathbb{R}$ be the entropy-maximizing asymptotic height function for $\left(R, h_{\partial R}\right)$. Let $g$ be the entropy-maximizing asymptotic height function for $\left(S,\left.f\right|_{\partial S}\right)$. Then $g=\left.f\right|_{S}$.

Proof. We will write $\operatorname{Ent}_{S}(),. \operatorname{Ent}_{R}(),. \operatorname{Ent}_{R \backslash S}($.$) for the entropy functionals of the various$ regions, and $A_{S}, A_{R}, A_{R \backslash S}$ for their areas. It is a direct consequence of (11) that

$$
A_{R} \operatorname{Ent}_{R}(f)=A_{S} \operatorname{Ent}_{S}(f)+A_{R \backslash S} \operatorname{Ent}_{R \backslash S}(f) .
$$

Define a height function $g^{*}$ on $R$ as follows:

$$
g^{*}(x)= \begin{cases}g(x) & \text { if } x \in S \\ f(x) & \text { if } x \in R \backslash S\end{cases}
$$

Observe that this function is a well-defined asymptotic height function because $g=f$ on the boundary of $S$. If $\operatorname{Ent}_{S}\left(\left.f\right|_{\partial S)}<\operatorname{Ent}_{S}(g)\right.$, then $\operatorname{Ent}_{R} f<\operatorname{Ent}_{R} g^{*}$, contradicting the fact that $f$ is the entropy maximizer on $R$. Thus $\operatorname{Ent}_{S}\left(\left.f\right|_{\partial S))}=\operatorname{Ent}_{S}(g)\right.$, and so $g=\left.f\right|_{S}$ is the unique entropy maximizer on $S$. 

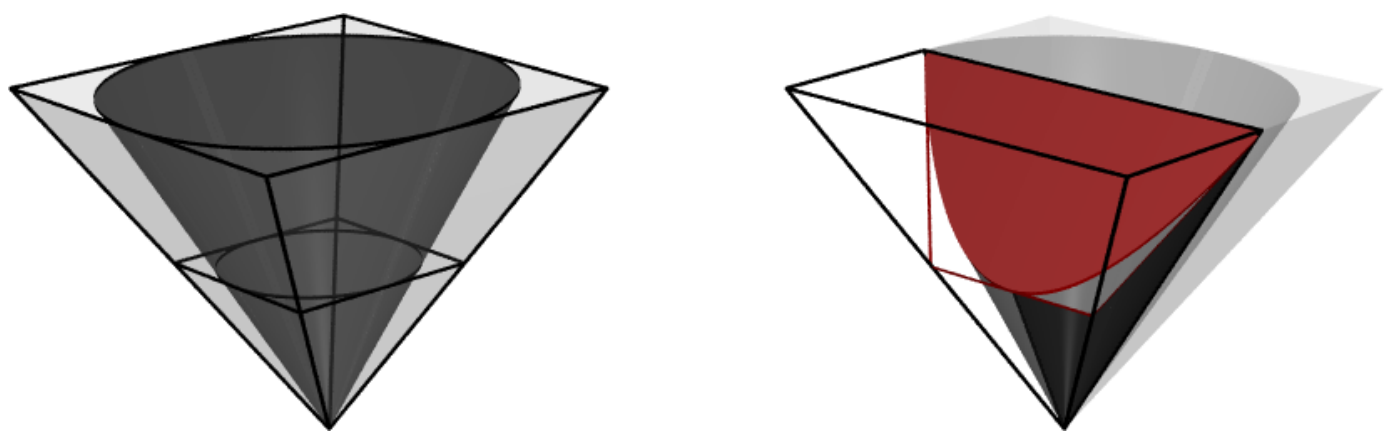

Figure 9: Space-time diagram for domino shuffling. Left image: the Aztec diamonds and their arctic circles. Right image: the half-hexagon and its arctic parabola.

Corollary 6.10 The limit shape of the normalized height function of the uniform random tiling of the Aztec half-diamond is the restriction of $G$ (from Theorem 6.7) to $y<\frac{1}{2}$.

Proof. Let

$$
\begin{aligned}
R & =[0,1] \times[0,1] & f(x, y)=G[x, y] \\
S & =[0,1] \times[0,1 / 2] & \left.g\right|_{\partial S}=\left.f\right|_{\partial S} ;
\end{aligned}
$$

That is, $R$ and $f$ describe the limit height function for the Aztec Diamond, and $S$ is the limiting region for the Aztec Half-Diamond, and $\left.g\right|_{\partial S}$ is its limiting height function by Equation (10). Lemma 6.9 now says that the limiting height function for the Aztec Half-Diamond $g$ coincides with $f_{S}$.

Corollary 6.11 The region of the uniformly tiled half-hexagon where particles (i.e. verticle lozenges) appear with positive probability is bounded by a parabola.

Indeed, one can show more: the normalized limiting height function of the half-hexagon is, up to a projective transformation, the same as that of the Aztec half-diamond. This transformation maps the boundary of one shape to the other, and it maps the arctic half-circle in the aztec diamond to the aforementioned parabola in the half-hexagon.

Proof. It was shown in Proposition 6.2 that Propp's algorithm gives a domino shuffle for the Aztec half-diamond. This shuffle defines a particle process $z_{i}^{j}(t)$. It is easy to check that particle process $z_{i}^{j}(t+i)$ is the same point process as that given by the half-hexagonshuffle: they have the same transition probabilities and the same boundary conditions. Indeed, both processes coincide with $X_{i}^{j}(t)$ of Equation (9) except at the center. To see that they coincide on the nose, one need only check that Propp's algorithm creates, deterministically, a line of equally spaced particles down the center of the Aztec halfdiamond at even times $t$, as is found in the half-hexagon, but this is immediate from the weights shown in Figure 7.

Consider the space-time diagram of the domino shuffle of an Aztec diamond, in which one draws the tiling produced at time $t$ parallel to the $x y$ plane, centered at the origin 
and at height $z=t$ (see Figure 9, left side). The diagram looks like a square pyramid; the arctic circles of the Aztec diamonds trace out a cone.

Consider next the space-time diagram of the domino shuffle of an Aztec Half-diamond (see Figure 9, right side). By Corollary 6.10, the region in which particles appear with positive probability is half of a cone, since the limiting height function coincides with that of the Aztec diamond at each value of $z$.

The half-hexagon process corresponds to the time-shifted slices parallel to one of the sides of this cone. As such the region in which $z_{i}^{j}(t)$ has a positive density of points is bounded by the intersection of a cone with a plane parallel to one of the cone's sides; this conic section is a parabola.

We remark that one can in fact write down the limiting height function in this way as well; it is precisely the image of the height function of Theorem 6.7 under the affine transformation which takes the rectangle $[0,1] \times[0,1 / 2]$ to the trapezoid with corners

$\{( \pm 1,0),( \pm(1 / 2), \sqrt{3} / 2)\}$. Moreover, domino shuffling on the Aztec half-diamond has a reasonably nice description: it turns out to coincide with a particular instance of Propp's Generalized Domino shuffling [Pro03] up to gauge transformation.

\section{$7 \quad$ Future work and open problems}

The reader will doubtless have noticed that this paper is a study of the phenomenology of one of the nicest, most special cases of the dimer model on planar bipartite graphs; naturally one should try to push the analysis to more general situations. For instance, a few other domino shuffles have been discovered on a variety of statistical mechanical models (see, for example, [YC10, BG09]) and it would be very interesting to study different space-time sections of them.

Taking slices through the cone in Figure 9 with varying slopes should yield a family of particle processes whose limit shapes are conic sections. Though this is a fairly trivial observation, we note that relatively few instances of the dimer model have known lowdegree algebraic limit shapes.

There is a general framework [BF08] for studying dynamics of the sort we have described in this paper, geared in particular to asymptotics. It appears that our model fits into this framework, but we have yet to determine whether the computations are tractable.

\section{References}

[BF08] Alexei Borodin and Patrik L. Ferrari. Anisotropic growth of random surfaces in 2+1 dimensions. arXiv:0804.3035, 2008.

[BG09] Alexei Borodin and Vadim Gorin. Shuffling algorithm for boxed plane partitions. Adv. Math., 220(6):1739-1770, 2009. 
[CEP96] Henry Cohn, Noam Elkies, and James Propp. Local statistics for random domino tilings of the Aztec diamond. Duke Math. J., 85(1):117-166, 1996.

[Ciu98] Mihai Ciucu. A complementation theorem for perfect matchings of graphs having a cellular completion. Journal of Combinatorial Theory, Series A, 81(1):34-68, 1998.

[CKP01] Henry Cohn, Richard Kenyon, and James Propp. A variational principle for domino tilings. J. Amer. Math. Soc., 14(2):297-346), 2001.

[DFR09] Philippe Di Francesco and Nicolai Reshetikhin. Asymptotic shapes with free boundaries. arXiv:0908.1630v1, 2009.

[EKLP92a] Noam Elkies, Greg Kuperberg, Michael Larsen, and James Propp. Alternating-sign matrices and domino tilings. I. J. Algebraic Combin., 1(2):111-132, 1992.

[EKLP92b] Noam Elkies, Greg Kuperberg, Michael Larsen, and James Propp. Alternating-sign matrices and domino tilings. II. J. Algebraic Combin., 1(3):219-234, 1992.

[EN11] Alexander Engstr om and Patrik Norén. Note: A bijection from poset cofibrations. manuscript, 2011.

[FF11] Benjamin Fleming and Peter Forrester. Interlaced particle systems and tilings of the aztec diamond. Journal of Statistical Physics, 142:441-459, 2011. 10.1007/s10955-011-0121-2.

[GV85] Ira Gessel and Gérard Viennot. Binomial determinants, paths, and hook length formulae. Adv. in Math., 58(3):300-321, 1985.

[GV89] I.M. Gessel and X. Viennot. Determinants, paths, and plane partitions. preprint, 132(197.15), 1989.

[JDLRV06] É. Janvresse, T. De La Rue, and Y. Velenik. A note on domino shuffling. The Electronic Journal of Combinatorics, 13(R30):1, 2006.

[Joh05a] Kurt Johansson. The arctic circle boundary and the Airy process. Ann. Probab., 33(1):1-30, 2005.

[Joh05b] Kurt Johansson. Non-intersecting, simple, symmetric random walks and the extended Hahn kernel. Ann. Inst. Fourier (Grenoble), 55(6):2129-2145, 2005.

[JPS98] William Jockusch, James Propp, and Peter Shor. Random domino tilings and the arctic circle theorem, 1998. arXiv:math.CO/9801068.

[Ken97] Richard Kenyon. Local statistics of lattice dimers. Ann. Inst. H. Poincaré Probab. Statist., 33(5):591-618, 1997.

[Ken04] Richard Kenyon. An introduction to the dimer model. In School and Conference on Probability Theory, ICTP Lect. Notes, XVII, pages 267-304 (electronic). Abdus Salam Int. Cent. Theoret. Phys., Trieste, 2004.

[KM59] Samuel Karlin and James McGregor. Coincidence probabilities. Pacific J. Math., 9:1141-1164, 1959. 
[KO07] Richard Kenyon and Andrei Okounkov. Limit shapes and the complex Burgers equation. Acta Math., 199(2):263-302, 2007.

[KOS06] Richard Kenyon, Andrei Okounkov, and Scott Sheffield. Dimers and amoebae. Ann. of Math. (2), 163(3):1019-1056, 2006.

[Lin73] Bernt Lindström. On the vector representations of induced matroids. Bull. London Math. Soc., 5:85-90, 1973.

[Nor10] Eric Nordenstam. On the shuffling algorithm for domino tilings. Electron. J. Probab., 15:no. 3, 75-95, 2010.

[Pro03] James Propp. Generalized domino-shuffling. Theor. Comput. Sci., 303(23):267-301, 2003.

[Rom09] Dan Romik. Arctic circles, domino tilings and square young tableaux. arXiv:0910.1636v1, 2009.

[Sta99] Richard P. Stanley. Enumerative combinatorics. Vol. 2, volume 62 of Cambridge Studies in Advanced Mathematics. Cambridge University Press, Cambridge, 1999. With a foreword by Gian-Carlo Rota and appendix 1 by Sergey Fomin.

[Thu90] William P. Thurston. Conway's tiling groups. Amer. Math. Monthly, 97(8):757-773, 1990.

[YC10] Benjamin Young and Cyndie Cottrell. Domino shuffling for the del Pezzo 3 lattice. arXiv:1011.0045, 2010. 